numbers; (2) the five closest hospitals geographically; (3) hospitals in the same administrative area; (4) a national comparison group. We also employed waiting time as an instrumental variable to account for possible effects on waiting times on patient numbers. We used three outcomes: overnight admissions; day-case admissions; and patients not attending for their booked appointments, and used a model segmented into three month periods after report publication in order to examine the range of short and longer term effects.

Results Two out of three of the hospitals we examined showed no difference in patient numbers at any point in the 12 months after negative reports were published. One of the hospitals showed a reduction in overnight patients for 3 months $(-12 \%, p=0.003)$ and 6 months $(-14 \%, p<0.001)$ after report publication, but patient levels returned to normal after this. Additional examination of patient numbers in advance of these reports coming out also did not change these results.

Conclusion The publication and dissemination of highly critical reports by a health care regulator does not appear to have resulted in patients' sustained avoidance of the hospitals involved. We only found an impact in one of the three hospitals we examined, and this only persisted for 6 months. This reminds us that simplistic assumptions regarding the power of information to drive patient numbers are unrealistic, and that reliance on these alone to drive quality improvement may be misguided.

\section{OP48 INCREASED MORTALITY ASSOCIATED WITH WEEKEND ADMISSION; A RESULT OF HIGHER SEVERITY OF ILLNESS AT THE TIME OF ADMISSION?}

doi:10.1136/jech-2012-201753.048

'P Paudyal, ${ }^{2}$ T Shepheard-Walwyn, ${ }^{3} \mathrm{C}$ Gericke. ${ }^{1}$ Peninsula College of Medicine and Dentistry, University of Plymouth, Plymouth, UK; '2lightfoot Solutions UK Limited, Lightfoot Solutions, Berkshire, UK; ${ }^{3}$ Peninsula College of Medicine and Dentistry, University of Plymouth, Plymouth, UK; ${ }^{4}$ Lightfoot Solutions UK Limited, Lightfoot Solutions, Berkshire, UK

Background A number of studies have shown that weekend admissions to hospital are associated with significantly higher mortality compared with weekday admissions. Previous studies have suggested that the observed variability in weekend-weekday mortality is a consequence of the quality of care provided in hospital. However, little information exists on whether the increased mortality associated with weekend admission is explained by the relative severity of illness at the time of admission.

Methods We performed a retrospective analysis of all emergency acute care admissions in England between April 2009 and March 2010 using Hospital Episode Statistics data. In-hospital mortality among patients who were admitted as emergency admission on weekends as compared to weekdays was analysed using the following measures: (1) overall deaths in hospital; (2) deaths in hospital within and after 3 days of admission (3) deaths in hospital among patients with 'higher risk' conditions and 'lower risk' conditions. The 'higher risk' conditions consisted of 103 Primary Diagnosis codes (ICD-10), which accounted for the 74\% of in-hospital deaths in the UK. The remaining Primary Diagnosis codes were categorised as 'lower risk' conditions.

Results There were 10,221,500 emergency hospital admissions during the two-year study period; $23.8 \%$ of the patients were admitted on weekends. A total of 391,938 patients died in hospital; a quarter of these died within 3 days of admission. Admission on weekends was associated with significantly higher in-hospital deaths as compared to weekday admission (Odds Ratio 1.11, 95\% CI 1.10-1.12). Mortality within 3 days of admission (early deaths) was also higher for patients admitted on weekends (OR 1.16, 95\%CI (1.14-1.17) and this difference persisted for both 'higher risk' and 'lower risk' conditions. However, when early deaths were excluded from the analysis, mortality was not affected by weekend admission.

Conclusion Higher mortality was seen among patients admitted on weekends compared to those admitted on weekdays. However, as the odds ratio for deaths after 3 days of admission was not affected by day of admission, it is probable that the weekend admission effect on mortality is at least in part due to higher severity of illness at the time of admission rather than purely being a consequence of poorer quality of in hospital care at the weekend, and this warrants further investigation.

\section{Thursday 13 September 2012}

\section{Parallel Session C}

\section{Socioeconomic Inequalities III}

\section{OP49 SOCIOECONOMIC STATUS AND LATE ADOLESCENT TRAJECTORIES OF SMOKING, DRINKING, AND PSYCHIATRIC DISTRESS}

doi:10.1136/jech-2012-201753.049

MJ Green, AH Leyland, H Sweeting, M Benzeval. MRC/CSO, Social \& Public Health Sciences Unit, Glasgow, Scotland

Background Problems with smoking, alcohol use and psychiatric distress usually develop in adolescence and often co-occur. These problems may be inter-related but their co-occurrence could result from a common cause such as socioeconomic disadvantage.

Methods The Twenty-07 Study includes self-reported data on smoking, alcohol use and psychiatric symptoms (GHQ-12) from 1,515 adolescents at approximate ages of 15 (baseline), 16 and 18 . Latent class analysis ( $n=1238$ with valid data) was used to identify distinct patterns of late adolescent development in smoking, alcohol use and psychiatric distress and relate these to various indicators of socioeconomic status (leaving school at 16, baseline area deprivation, and baseline parental measures of social class, education, housing tenure, income, employment status and family structure) whilst adjusting for gender.

Results Four latent classes were identified: 1) a low-risk class with low levels of smoking at all ages, low early drinking, moving towards regular drinking at age 18, and low levels of psychiatric symptoms; 2) a class with similar patterns for smoking and drinking but with high levels of psychiatric distress; 3) a class which engaged with alcohol earlier, many of whom were heavy drinkers by age 18 , and had medium levels of distress; and 4) a high-risk class who engaged early with both smoking and drinking, most of whom were heavy smokers by age 18 , and had medium and increasing levels of distress. In unadjusted analyses most indicators of socioeconomic disadvantage were associated with raised odds of membership in the highrisk class (reference group: low-risk class). With mutual adjustment for gender and all socioeconomic variables, those leaving school at 16 (OR 6.01; 95\% CI 3.93-9.17) and in rented accommodation (OR $1.74 ; 1.19-2.56)$ still had significantly raised odds of membership in the high-risk class relative to those staying in school or in owneroccupied accommodation. Rented accommodation was protective for membership in the class with drinking but not smoking problems (OR 0.51; 0.30-0.86) and those in the most deprived areas were less likely to be in the class with psychiatric distress only (OR 0.37; 0.17-0.83) than those in more affluent areas.

Conclusion Socioeconomic disadvantage is implicated as a common cause of smoking, alcohol use, and psychiatric distress. Relationships with socioeconomic status are complex however and depend on the combination of outcomes, for example, trajectories with drinking but not smoking problems and those with psychiatric 
distress alone were more likely at the higher end of the socioeconomic distribution.

\section{OP50 ALCOHOL INTAKE, DRINKING BEHAVIOUR AND DRINKING PATTERNS AS PREDICTORS OF EMPLOYMENT STATUS IN WORKING-AGE MEN IN IZHEVSK, RUSSIA}

doi:10.1136/jech-2012-201753.050

S A Cook, D A Leon. Faculty of Epidemiology and Population Health, London School of Hygiene \& Tropical Medicine, London, UK

Background Hazardous alcohol consumption and unemployment are both associated with high mortality in Russia. Unemployment adversely affects mental and physical health. The relationship between alcohol and employment is complex as alcohol consumption may lead to unemployment but unemployment may also increase hazardous consumption. Very few longitudinal studies have investigated the effects of alcohol use on employment status. The objectives of this study were to investigate alcohol intake, drinking behaviour and drinking patterns as predictors of employment status in a longitudinal study of working-age men in Izhevsk, Russia.

Methods Participants were 1217 men aged 25-54 resident in Izhevsk, Russia in regular paid employment at baseline (2003-6) who were followed up and re-interviewed (2008-9). Alcohol use was measured by total volume of ethanol consumed from beverage alcohol, drinking behaviour (abstainers, beverage alcohol drinkers with no problem drinking behaviour, beverage alcohol drinkers with problem drinking behaviour and non-beverage alcohol drinkers (e.g. eau de cologne)), and three aspects of drinking pattern (drinking spirits without food, drinking alone and drinking before noon). Problem drinking was defined as twice weekly or more frequency of hangover, excessive drunkenness, sleeping in clothes because of drunkenness or one or more episodes of zapoi in the past year (defined as continuous drunkenness of two or more days during which participants were withdrawn from normal life). Logistic regression was used to investigate whether alcohol use at baseline predicted employment status at follow up after adjusting for sociodemographic variables.

Results Total volume of ethanol from beverage alcohol at baseline did not predict whether men were still in regular paid employment at follow-up ( $\mathrm{P}$ value for linear trend $=0.21$ ). Problem drinkers (adjusted odds ratio 2.82 95\% CI 1.23, 6.46) and non-beverage alcohol drinkers (adjusted odds ratio 2.42 (95\% CI 1.18, 4.93) were more likely not to be in regular employment at follow-up than non-problem beverage alcohol drinkers. Among drinkers drinking spirits without food (adjusted odds ratio 2.13 95\% CI 1.28, 3.54), drinking alone (adjusted odds ratio $1.6595 \%$ CI 1.09, 2.50) and drinking before noon (adjusted odds ratio 2.49 95\% CI 1.65, 3.78) all predicted employment status at follow-up.

Conclusion Drinking behaviour and drinking patterns predicted future employment status in a sample of working-age men in Izhevsk Russia. However the volume of alcohol consumed per year did not, suggesting how alcohol is consumed and whether it leads to problem behaviour is more important than the overall amount consumed when considering the impact of alcohol on employment.

\section{OP51 MATERNAL EMPLOYMENT AND SOCIO-EMOTIONAL BEHAVIOUR AT AGE 7: FINDINGS FROM THE UK MILLENNIUM COHORT STUDY}

doi:10.1136/jech-2012-201753.051

SC Hope, A Pearce, C Law. MRC Centre of Epidemiology for Child Health, UCL Institute of Child Health, London, UK

Background Maternal employment in the UK has increased significantly in recent years, but evidence of its relationship with child socio-emotional behaviour is mixed. Given the policy importance of both family employment and children's wellbeing, this relationship needs to be better understood. This study seeks to investigate whether cumulative exposure to maternal employment is associated with socio-emotional behaviour at age 7 .

Methods The study comprises a longitudinal analysis of the United Kingdom Millennium Cohort Study (MCS), based on 10,723 singleton children who participated in all four sweeps of the study (at ages 9 months, 3,5 and 7 years). Socio-emotional behaviour was measured using total Strengths and Difficulties Questionnaire (SDQ) scores, dichotomised using an established cut-off for borderline / abnormal behaviour. Risk ratios (RR) were estimated using Poisson regression for borderline / abnormal SDQ scores at age 7 according to cumulative maternal employment over four sweeps of the MCS (at 9 months, 3, 5 and 7 years), unadjusted (uRR) and adjusted (aRR) for confounders (age at first live birth, ethnicity, family size, lone parenthood, and maternal psychological distress, all recorded at 9 months). In order to disentangle whether there was a differential impact of earlier and current employment, additional analyses estimated the risk associated with employment up to age 5 years, adjusting for employment at age 7.

Results The risk of borderline/abnormal scores decreased linearly by number of MCS sweeps that mothers were in employment; after adjustment for confounders the gradient attenuated but remained significant. Children growing up in households where mothers were employed in all MCS sweeps were significantly less likely to display borderline / abnormal behaviour at age 7 (compared to never in paid employment): $\mathrm{uRR}=0.37$ (0.32-0.44); $\mathrm{RRR}=0.68(0.56-0.81)$. Analyses differentiating early employment (between ages 9 months and 5 years) and current employment (at age 7) suggest that the cumulative impact of prior non-employment may be ameliorated once a mother gains employment. After adjusting for current employment and confounders, there was no increased risk of borderline / abnormal socio-emotional behaviour associated with non-employment in all earlier sweeps up to age 5 compared to being in paid employment continuously (baseline): $\mathrm{aRR}=1.01(0.82-1.23)$.

Conclusion This longitudinal analysis supports an association between maternal employment and child socio-emotional behaviour. Possible explanations and implications will be discussed.

\section{OP52 LONG-TERM EXPOSURE TO INCOME INEOUALITY: IMPLICATIONS FOR PHYSICAL AND MENTAL HEALTH AT OLDER AGES}

doi:10.1136/jech-2012-201753.052

R de Vries, D Blane, G Netuveli. Primary Care and Public Health, Imperial College London, London, UK

Background The 'inequality hypothesis' proposes that higher levels of societal income inequality have a detrimental effect on both physical and mental health. Previous studies have provided only mixed support for this hypothesis, particularly among older people. However, by using only contemporary income inequality estimates, or estimates from a single lag period, the majority of previous studies have not accounted for people's continued exposure to income inequality over the long-term. In this study, we addressed this problem by examining the association between older people's experience of inequality over an extended period and their subsequent health.

Methods The data for this project were taken from three comparable nationally representative surveys (covering 16 countries) of the health and circumstances of older people; the English Longitudinal Study of Ageing, the Survey of Health and Retirement in Europe, and the U.S. Health and Retirement Study. Standardised estimates of national income inequality from 1960-2006 were taken from the Standardised World Income Inequality Database. We used multilevel regression methods to model the association between average 